\title{
INFLUENCIA DE LOS ESTILOS PARENTALES EN LA ADICCIÓN AL INTERNET EN ALUMNOS DE SECUNDARIA DEL PERÚ
}

\author{
INFLUENCE OF PARENTING STYLES ON ADDICTION TO INTERNET IN \\ PERUVIAN SECONDARY STUDENTS
}

\author{
Maria Matalinares C., Gloria Diaz A., Ornella Raymundo V., Deyvi Baca R., Erika Fernández A., Joel Uceda E., \\ Victor Leyva O., Elsa Sánchez Y., Nayda Villavicencio C., Juan Yaringaño L., Jenny Torre M., \\ Moira Encalada D., Ailin Diaz V. \\ Universidad Nacional Mayor de San Marcos, Lima, Perú \\ (RECIBIDO 15-06-2013, AcEPTAdo 21-08-2013)
}

\begin{abstract}
RESUMEN
El presente estudio tuvo como objetivo establecer la influencia de los estilos parentales en la adicción al Internet. Se evaluó a 2370 adolescentes escolares de tercero a quinto de secundaria de 14 ciudades representativas de la costa, sierra y selva del Perú, con edades entre 13 y 18 años, a quienes se aplicó el cuestionario de estilos parentales de Parker, et al. (1997) adaptado a nuestra realidad para el presente estudio y el test de adicción al Internet de Young (1998) adaptado a la realidad por Raymundo, Matalinares y Baca (2013, en prensa). Los resultados mostraron que los estilos parentales disfuncionales (abuso e indiferencia) influyen en la adicción al Internet. Se obtuvo una mayor incidencia de adolescentes con adicción al Internet en la costa, mientras que en la sierra hay una mayor percepción de estilos parentales disfuncionales. También se halló que los hogares monoparentales evidencian un mayor uso de estilos parentales disfuncionales (indiferencia, abuso y sobreprotección).
\end{abstract}

Palabras clave: Adicción, estilos parentales disfuncionales, indiferencia, abuso, sobreprotección

\begin{abstract}
Present study aimed to establish influence of parenting styles on addiction to Internet. 2370 teenage students from third to fifth grades of secondary school were evaluated. They belonged to 14 representative cities of coast, highlands and forest Peruvian regions and aged 13 to 18 years. Parenting Styles Questionnaire (Parker et al. 1997), adapted to our reality for the present study, and Addiction to Internet Test (Young, 1998), also adapted to our reality by Raymundo, Matalinares and Baca (2013, pending publication) were the instruments applied. Results showed that dysfunctional parenting styles (abuse and indifference) influenced on addiction to Internet. Greater incidence of teenage students addicted to Internet was observed in the coast while greater perception of dysfunctional parenting styles was observed in highlands. Dysfunctional parenting styles (indifference, abuse, overprotection) on one-parent family homes were also found.
\end{abstract}

Keywords: Addiction, dysfunctional parenting styles, indifference, abuse, overprotection

\footnotetext{
* Docente Principal UNMSM. E-mail:
} 


\section{INTRODUCCIÓN}

El estilo parental puede ser entendido como una constelación de actitudes acerca del niño, que le son comunicadas y que, en conjunto, crean un clima emocional en el que se ponen de manifiesto los comportamientos de los padres. Estos comportamientos incluyen tanto las conductas a través de las cuales los padres desarrollan sus propios deberes de paternidad (prácticas parentales) como cualquier otro tipo de comportamientos como gestos, cambios en el tono de voz, expresiones espontáneas de afecto, etc. (Darling y Steinberg, 1993 citados por Raya 2008). Autores como Esteves, Murgui, Musiti y Moreno hacia el 2008, explican que la familia, por una parte, representa el eje central del ciclo vital de acuerdo con el cual transcurre la existencia de las personas: se trata de una institución social fundamentada en relaciones afectivas y desde la que el niño y adolescente aprehende los valores, creencias, normas y formas de conducta apropiadas para la sociedad a la que pertenece. Este proceso tiene lugar en un ambiente o clima social, que se define como el ambiente percibido e interpretado por los miembros que integran una organización -en este caso la familia- y que, a su vez, ejerce una importante influencia en el comportamiento de los integrantes de ese contexto, así como en su desarrollo social, físico, afectivo e intelectual (Martínez, 1996 citado por Esteves, Murgui, Musitu y Moreno (2008).

La asociación entre el estilo parental o vincular y trastornos psicopatológicos puede observarse desde etapas muy precoces y a lo largo de los diferentes períodos del desarrollo del ser humano. Melis, Dávila, Ormeño y cols. (2001, citados en Vergara, 2005) sostienen que la repetición constante de un patrón vincular es necesaria para la existencia de una relación vincular segura; al mismo tiempo, la presencia de un patrón distorsionado tiene influencias en la personalidad desde que esta se instaura. En tal sentido, Parker, Tupling y Brown (1979) consideran que hay una significativa asociación entre parentalidad disfuncional (padres poco afectuosos y sobreprotectores) y los trastornos psiquiátricos, estos autores identificaron 3 tipos de vínculos parentales disfuncionales: indiferencia, abuso y sobreprotección.

1. Indiferencia: Este estilo parental se caracteriza por la ausencia de exigencias y de cumplimiento de responsabilidades, falta de estructuración, control y apoyo. Estos padres generalmente desvían sus responsabilidades paternas hacia otras figuras como el colegio u otros familiares; sus hijos presentan problemas de conducta, el ambiente familiar suele ser desorganizado y son altamente vulnerables a la ruptura familiar. (Steinber, Blatt-Eisengart \& Cauffman, 2006; Raya, 2008).

2. Abuso: Es un patrón estricto de crianza en la que los padres imponen las reglas para sus hijos, esperan una obediencia estricta y se basan en el poder que estos tienen como padres (Baumrind, 2005), se generan castigos físicos y verbales.

3. Sobreprotección: Este estilo se caracteriza por combinar bajo control y exigencia con poca sensibilidad hacia las necesidades de los hijos, muchas veces pueden ser crueles y no establecen normas, no se muestran firmes frente a sus hijos, no esperan acciones maduras de sus hijos. La comunicación no es efectiva y suele ir en una sola dirección de padre al hijo (Baumrind, 2005). 
En la actualidad, es posible afirmar que la calidad de los cuidados que recibe un niño en sus primeros años es de suma importancia para su futura salud mental. Es decir, que las relaciones que mantuvieron durante su infancia con sus padres van a influir en las relaciones que mantenga como actual cuidador con su propio hijo (Soares, 1996; citado en Fourment, 2009) generando ciertos patrones o tendencias en sus modos de relacionarse.

En el extranjero se han realizado una serie de investigaciones sobre los estilos parentales siguiendo la tipología de los padres y su influencia en el desarrollo de los hijos (Blake, Clements y Vazsonyi, 2004; Lamborn, Mounts, Steinberg y Dornbusch, 1991; Pellerin, 2004, Cakir y Aydin, 2005; Chao, 2001; Chao, 2001; Endicott y Liossis, 2005; Glasgow, Musitu y García, 2001; Shek, Lee y Chan, 1998; Sorkhabi, 2005) citados por Vallejo, Osorno y Mazadiego (2008). En el Perú son escasas las investigaciones sobre estilos parentales realizadas: Canales (2000), Gonzales-Vigil (2008), Aponte (2009), Yana (2009), Arenas (2009), Jarrin (2011).

Ahora bien, respecto de la variable adicción al Internet es importante señalar que el Internet es una herramienta tecnológica que ha surgido en los últimos 20 años y ha permitido que los individuos se intercomuniquen en todas partes del mundo ofreciendo últimos avances en ciencia y tecnología. Es desde el año 1996 con la publicación de un caso de Kimberly Young, sobre una ama de casa con una vida hogareña satisfecha, al cabo de tres meses de descubrir las salas de charlas (chat), empezó a dedicar hasta 60 horas por semana en esta conectada en línea. Describió tener una adicción al medio "como si lo tuviese al alcohol". Al cabo de un año de adquirir su propio ordenador para su domicilio, hacía caso omiso de las tareas domésticas, había renunciado a sus actividades sociales que solía disfrutar y se había llegado a separar de sus dos hijas adolescentes y su esposo después de 17 años (Aboujaude, 2010; 85).

La citada autora basada en este y otros casos comienza a estipular criterios diagnósticos para la adicción al Internet, considerando, en un primer momento, los criterios diagnósticos de dependencia a las sustancias para luego asociarlo al juego patológico del DSM-IV, es bajo estos criterios que elabora el test de adicción al Internet en 1998. Sin embargo, en la actualidad el DSM-V lo ha consignado como el trastorno impulsivo compulsivo por el uso de Internet (Beard y Wolf, 2001, citado en Block, 2008). Conceptualmente, el diagnóstico es un trastorno impulsivo compulsivo por el uso de Internet (Dell'Osso, Altamura, Allen, Marazziti, y Hollander, 2006, citados en Young, y Nabuco, 2011). Todas 1 as variantes comparten los siguiente cuatro componentes: los cuales se describen como:

a. El uso excesivo: Está asociado con una pérdida del sentido del tiempo, como una característica impulsiva del inicio del problema, donde la persona no logra controlar la necesidad de estar conectado al Internet, dejando de lado actividades sociales y familiar por pensar y estar conectado en línea, pasando más tiempo del que se había programado. 


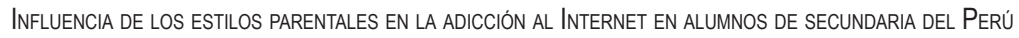

b. La tolerancia: Es la necesidad de un mejor equipamiento informático, un nuevo programa, o más horas de uso, como una reacción compulsiva para aliviar la ansiedad de no estar conectado, o ansiedad por situaciones estresantes de la vida cotidiana, las mismas que son reemplazadas por el uso del Internet.

c. La retirada o abstinencia: Se aprecian sentimientos de ira, ansiedad, y/o depresión cuando la computadora es inaccesible, interfiriendo en sus pensamientos, emociones y motivaciones, reduciendo el intervalo de tiempo de un acceso a otro a Internet.

d. Consecuencias negativas: Se aprecian argumentos, mentiras, bajo rendimiento, aislamiento social, fatiga y descuido en las actividades domésticas como consecuencia de estar conectado al Internet.

A nivel mundial se han realizado una serie de investigaciones sobre la adicción al Internet: Kraut y cols. (1998), Young y Rodgers (1998) Arai, González, Morales, Gutiérrez y Tettley (2008), Yen, Ko, Yen, Chen, Chung y Chen (2008), Herrera, Pacheco, Palomar y Zavala (2010).

En el Perú investigadores como Cruzado, Matos, Kendall (2006), Quispe (2011) Lam, Contreras, Mori, Nizama y cols. (2011) y Matalinares y cols. (2012) investigaron la adicción al Internet.

En base a lo expuesto, la presente investigación intenta abordar la influencia de los estilos parentales disfuncionales sobre la adicción al Internet, sobre todo se abordarán los estilos parentales de padre y madre en forma separada.

\section{Objetivo general}

Identificar cómo influyen los estilos parentales en la adicción al Internet en estudiantes de secundaria del Perú.

\section{Objetivos específicos}

1. Desarrollar el diagnóstico de la adicción al Internet de los estudiantes de secundaria del Perú.

2. Desarrollar el diagnóstico de los estilos parentales de los estudiantes de secundaria del Perú.

3. Adaptar y baremar el test de estilos parentales para los adolescentes de educación secundaria del Perú.

4. Determinar las diferencias en la adicción al Internet en función de variables como el género, ubicación geográfica, edad cronológica en estudiantes de educación secundaria del Perú.

5. Determinar las diferencias en los estilos parentales en función de variables como el género, ubicación geográfica, y edad cronológica en estudiantes de educación secundaria del Perú. 


\section{MÉTODO}

En concordancia con las hipótesis formuladas se empleó un tipo de investigación Sustantiva - Explicativa, ya que está orientada al descubrimiento de los factores causales que han podido incidir o afectar la ocurrencia de un fenómeno (Sánchez y Reyes, 2009).

El diseño de investigación utilizado en el presente estudio fue no experimental, transeccional-causal. La muestra estuvo constituida por 2370 adolescentes escolares de tercero, cuarto y quinto de secundaria, pertenecientes a 14 ciudades representativas del Perú: Tacna, Lima Metropolitana, Trujillo, Tumbes, Huanta, Cerro de Pasco, Cuzco, Huánuco, Huaraz, Huancavelica, Huancayo, Bagua Grande, Moyobamba y Pucallpa. Todas ellas agrupadas en las tres regiones naturales (costa, sierra y selva) principalmente los escolares pertenecían a la región natural Sierra en un 51.4\%, seguido de la región Costa por un $28 \%$ y, finalmente, la región Selva quedó representada por un $20.8 \%$ del total de la muestra. Los adolescentes escolares tuvieron edades entre los 13 y 18 años. Destacándose un predominio del $31.7 \%$ adolescentes con 15 años, seguido por un $26.1 \%$ que poseía 14 años. Asimismo, se detectó un menor porcentaje de adolescentes de 18 años y 13 años con porcentajes de $1.9 \%$ y $8.2 \%$, respectivamente. Se apreció un ligero predominio de las adolescentes mujeres. Conformando el 52.4\%; mientras que un $47.6 \%$ fueron varones.

Técnica e Instrumentos: Dado el carácter de los fenómenos a estudiar se utilizaron:

La técnica psicométrica y de observación indirecta. Los instrumentos utilizados fueron:

1. Escala de estilos parentales MOPS (Black Dog Institute, 2007; Parker, Roussos, Hadzi, Mitchell, Wilhelm y Austin, 1997). Instrumento diseñado para capturar los riesgos del comportamiento de educación en los 16 primeros años de la vida de un niño. Los participantes evalúan cada ítem como una descripción de su madre $\mathrm{y}$, sobre una forma separada, el comportamiento de su padre hacia ellos en sus 16 primeros años. El MOPS incluye tres escalas: indiferencia (seis ítems), abuso (cinco ítems), y sobrecontrol (cuatro ítems). La prueba tiene la consistencia interna de 0.829 en la dimensión de estilos parentales de los padres, mientras que en la consistencia interna de la dimensión de los estilos parentales de las madres es de 0.781, ambas aceptables y confiables. La teoría que sostiene el MOPS está basada en Bowlby (1977, como se cita en Melis, Dávila, Ormeño, Vera, Greppi y Gloger, 2001) la definición de educación anómala donde los padres fallan en proporcionar el cuidado por ser insensibles, el menosprecio, el rechazamiento, o teniendo la sobreprotección excesiva o el control. Estos comportamientos paternales y actitudes son considerados como una predisposición en un niño al desorden psiquiátrico así como a interacciones disfuncionales sociales y/o emocionales en la edad adulta (Parker et al., 1997).

2. Test de adicción al Internet propuesto por Young (1996) estandarizado por Raymundo, Matalinares y Baca (2013, en prensa). Su administración puede ser individual o 


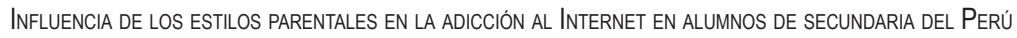

colectiva, el tiempo de aplicación es en promedio 20 minutos. El test evalúa los indicadores de adición al Internet distribuidos en cuatro componentes: el uso excesivo, la retirada, tolerancia y consecuencias negativas, la tipificación se realizó mediante baremos nacionales según el sexo y lugar de procedencia (costa, sierra y selva).

La confiabilidad total del test es de 0.870 . En el análisis factorial exploratorio obtuvieron cuatro componentes: tolerancia, uso excesivo, retirada y consecuencias negativas, con una varianza de $49.57 \%$. Estos componentes fueron ratificados mediante el análisis factorial confirmatorio.

El test de adicción al Internet adaptado al Perú, contempla los criterios diagnósticos del DSM -V para el trastorno impulsivo compulsivo por el uso de Internet, presenta los siguientes cuatro componentes:

a. El uso excesivo: Está asociado con una pérdida del sentido del tiempo, como una característica impulsiva del inicio del problema, donde la persona no logra controlar la necesidad de estar conectado al Internet, dejando de lado actividades sociales y familiar por pensar y estar conectado en línea, pasando más tiempo del que se había programado.

b. La tolerancia: Es la necesidad de un mejor equipamiento informático, un nuevo programa, o más horas de uso, como una reacción compulsiva para aliviar la ansiedad de no estar conectado, o ansiedad por situaciones estresantes de la vida cotidiana, las mismas que son reemplazadas por el uso del Internet.

c. La retirada o abstinencia: Se aprecian sentimientos de ira, ansiedad, y/o depresión cuando la computadora es inaccesible, interfiriendo en sus pensamientos, emociones y motivaciones, reduciendo el intervalo de tiempo de un acceso a otro a Internet.

d. Consecuencias negativas: Se aprecian argumentos, mentiras, bajo rendimiento, aislamiento social, fatiga y descuido en las actividades domésticas como consecuencia de estar conectado al Internet.

\section{RESULTADOS}

\section{Análisis descriptivo del acceso y uso del Internet}

Dentro de la Tabla 1, se destaca que los adolescentes escolares utilizan, en ambos sexos, como medio de acceso al Internet una cabina pública: $50.8 \%$ de varones y $53.5 \%$, mujeres. 
Maria Matalinares C., Gloria Diaz A., Ornella Raymundo V., Deyvi Baca R., Erika Fernández A.

Tabla 1. Distribución del acceso al Internet en adolescentes escolares según sexo (varones y mujeres)

\begin{tabular}{lcccc}
\hline \multirow{2}{*}{ Acceso al Internet } & \multicolumn{2}{c}{ Varones } & \multicolumn{2}{c}{ Mujeres } \\
& Frecuencia (f) & Porcentaje (\%) & Frecuencia (f) & Porcentaje (\%) \\
\hline Casa & 316 & 28.0 & 408 & 32.8 \\
Cabina & 573 & 50.8 & 665 & 53.5 \\
Casa y cabina & 238 & 21.1 & 170 & 13.7 \\
\hline Total & 1127 & 100.0 & 1243 & 100.0 \\
\hline
\end{tabular}

De acuerdo al uso del Internet, se aprecia en la Tabla 2 que el $53.8 \%$ de los adolescentes y $60.3 \%$ de las adolescentes permanecen al menos de 01 hora diaria; seguido del uso entre 01 a 05 horas diarias en varones y mujeres representado por el $40.6 \%$ y $35.4 \%$, respectivamente.

Tabla 2. Distribución del tiempo de uso del Internet en adolescentes escolares según sexo (varones y mujeres)

\begin{tabular}{lcccc}
\hline \multirow{2}{*}{$\begin{array}{l}\text { Tiempo de uso del Internet } \\
\text { (diaria) }\end{array}$} & Frecuencia $(f)$ & Porcentaje (\%) & Frecuencia (f) & Porcentaje (\%) \\
\hline Menos de 01 hora & 606 & 53.8 & 750 & 60.3 \\
Entre 01 hasta 05 horas & 458 & 40.6 & 440 & 35.4 \\
Desde 05 hasta 10 horas & 51 & 4.5 & 31 & 2.5 \\
Más de 10 horas & 12 & 1.1 & 22 & 1.8 \\
\hline Total & 1127 & 100.0 & 1243 & 100.0 \\
\hline
\end{tabular}

Siguiendo la Tabla 3, se tiene que de acuerdo al contenido accedido por Internet se encuentra en los varones en mayor medida las redes sociales $(21.8 \%)$, seguido por redes sociales y actividades escolares (19\%), y juegos online, redes sociales y contenido sexual (19\%). En contraste, la mujeres accedieron a redes sociales y actividades escolares (35.6\%), seguido de actividades escolares (25.5\%).

Tabla 3. Distribución del tipo de contenido accedido por Internet en adolescentes escolares según sexo (varones y mujeres)

\begin{tabular}{lcccc}
\hline \multirow{2}{*}{$\begin{array}{l}\text { Tipo de contenido accedido por } \\
\text { Internet }\end{array}$} & \multicolumn{2}{c}{ Varones } & \multicolumn{2}{c}{ Mujeres } \\
\hline Juegos online & Frecuencia (f) & Porcentaje (\%) & Frecuencia (f) & Porcentaje (\%) \\
Contenido sexual & 37 & 6.3 & 45 & 3.6 \\
Redes sociales & 246 & 3.3 & 20 & 1.6 \\
Actividades escolares & 134 & 11.9 & 229 & 18.4 \\
Juegos online y redes sociales & 128 & 11.4 & 317 & 25.5 \\
Juegos online y actividades & 83 & 7.4 & 30 & 3.2 \\
escolares & & & 39 & 3.1
\end{tabular}




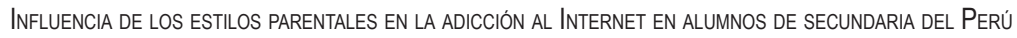

\begin{tabular}{lcccc} 
Redes sociales y actividades & 214 & 19.0 & 442 & 35.6 \\
$\begin{array}{l}\text { escolares } \\
\text { Juegos online, redes sociales y }\end{array}$ & 214 & 19.0 & 111 & 8.9 \\
\hline contenido sexual & 1127 & 100.0 & 1243 & 100.0 \\
\hline Total & & & \\
\hline
\end{tabular}

\section{Análisis descriptivo de los estilos parentales}

En la Tabla 4 se tiene que en el estilo parental del padre de tipo indiferencia es percibido en un nivel alto por el $15.88 \%$ de los adolescentes y el $18.26 \%$ de las adolescentes. El estilo parental de abuso es percibido en un nivel alto por el $18.73 \%$ y $14.88 \%$ de varones y mujeres, respectivamente. Y, en estilo parental de sobreprotección es percibido en un nivel alto por $8.87 \%$ de los adolescentes y $10.38 \%$ de las adolescentes.

Tabla 4. Distribución por niveles de la sub-variable, estilos parentales del padre, percibido por los adolescentes escolares según sexo (varones y mujeres)

\begin{tabular}{ccccc}
\hline \multicolumn{2}{c}{ Varones } & \multicolumn{3}{c}{ Mujeres } \\
Estilos Parentales & Frecuencia (f) & Porcentaje (\%) & Frecuencia (f) & Porcentaje (\%) \\
\hline Indiferencia & 179 & 15.88 & 227 & 18.26 \\
\hline Alto & 948 & 84.12 & 1016 & 81.74 \\
Medio & 0 & 0.00 & 0 & 0.00 \\
Bajo & 201 & & 185 & 14.88 \\
\hline Abuso & 926 & 17.83 & 1058 & 85.12 \\
\hline Alto & 02.17 & 0 & 0.00 \\
\hline Medio & 0 & 0.00 & & 10.38 \\
\hline Bajo & 100 & & 129 & 73.93 \\
\hline Alto & 875 & 8.87 & 919 & 15.69 \\
\hline Motal & 152 & 77.64 & 195 & 100.0 \\
\hline
\end{tabular}

Con respecto a la Tabla 5 se muestran los estilos parentales de la madre. El estilo parental de indiferencia es percibido en un nivel alto por los adolescentes y las adolescentes en un $16.33 \%$ y $18.18 \%$, respectivamente. Asimismo, se encontró que estilo parental de abuso es percibido en un nivel alto por $15 \%$ y $14.32 \%$ de varones y mujeres, respectivamente. Y, el estilo parental sobreprotección es percibido en un nivel alto por el $19.61 \%$ de los adolescentes y $18.02 \%$ de las adolescentes. 
Maria Matalinares C., Gloria Diaz A., Ornella Raymundo V., Deyvi Baca R., Erika Fernández A.

Tabla 5. Distribución por niveles de la sub-variable, estilos parentales de la madre, percibido por los adolescentes escolares según sexo (varones y mujeres)

\begin{tabular}{ccccc}
\hline \multirow{2}{*}{ Estilos Parentales } & \multicolumn{2}{c}{ Varones } & \multicolumn{2}{c}{ Mujeres } \\
\cline { 3 - 5 } & Frecuencia (f) & Porcentaje (\%) & Frecuencia (f) & Porcentaje (\%) \\
\hline Indiferencia & 184 & 16.33 & 226 & 18.18 \\
\hline Alto & 943 & 83.67 & 1017 & 81.82 \\
Medio & 0 & 0.00 & 0 & 0.00 \\
\hline Bbajo & 169 & 15.00 & 178 & 14.32 \\
\hline Alto & 958 & 85.00 & 1065 & 85.68 \\
Medio & 0 & 0.00 & 0 & 0.00 \\
\hline Bajo & & & & 18.02 \\
\hline Sobreprotección & & & 224 & 59.61 \\
\hline Alto & 221 & 19.61 & 741 & 22.37 \\
\hline Medio & 694 & 61.58 & 278 & 100.0 \\
\hline Botal & 212 & 18.81 & 1243 &
\end{tabular}

\section{Análisis descriptivo de la adicción al Internet}

Se observa en la Tabla 6, los niveles de la Adicción al Internet. Los varones se ubicaron en un nivel muy alto el $11.27 \%$; en contraste, el $12.39 \%$ de mujeres se hallo en dicho nivel.

Tabla 6. Distribución por niveles de la variable Adicción al Internet en adolescentes escolares según sexo (varones y mujeres)

\begin{tabular}{lcccc}
\hline \multirow{2}{*}{ Adicción al Internet } & \multicolumn{2}{c}{ Varones } & \multicolumn{2}{c}{ Mujeres } \\
& Frecuencia (f) & Porcentaje (\%) & Frecuencia (f) & Porcentaje (\%) \\
\hline Muy Alto & 127 & 11.27 & 154 & 12.39 \\
Alto & 170 & 15.08 & 220 & 17.70 \\
Medio & 539 & 47.83 & 529 & 42.56 \\
Bajo & 186 & 16.50 & 172 & 13.84 \\
Muy Bajo & 105 & 9.32 & 168 & 13.52 \\
\hline Total & 1127 & 100.0 & 1243 & 100.0 \\
\hline
\end{tabular}

Análisis inferencial

Se aprecia en la Tabla 7 que el p-valor para ambos instrumentos fue inferior al nivel de significación $(\square=0.05)$. Por ello se utilizarán pruebas no paramétricas para los posteriores análisis inferenciales. 


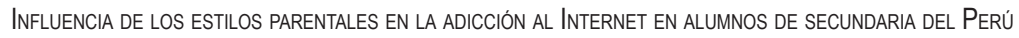

Tabla 7. Normalidad del Test de Adicción al Internet y Escala de Estilos Parentales a través de la prueba de Kolmogorov-Smirnov en adolescentes escolares $(n=2370)$

\begin{tabular}{|c|c|c|c|c|c|c|c|}
\hline \multirow{2}{*}{$\begin{array}{l}\text { Instrumentos / } \\
\text { Factores }\end{array}$} & \multicolumn{2}{|c|}{ Parámetros } & \multicolumn{3}{|c|}{ Diferencias Extremas } & \multirow{2}{*}{$\begin{array}{l}\text { Kolmogorov- } \\
\text { Smirnov Z }\end{array}$} & \multirow{2}{*}{$\begin{array}{c}\text { Sig. } \\
\text { (bilateral) }\end{array}$} \\
\hline & Media & $\begin{array}{l}\text { Desviación } \\
\text { Estándar }\end{array}$ & Absoluto & Positivo & Negativo & & \\
\hline $\begin{array}{l}\text { Escala de Estilos } \\
\text { Parentales }\end{array}$ & 9.65 & 6.803 & 0.147 & 0.147 & -0.078 & 7.145 & 0.000 \\
\hline Padre & 3.40 & 3.514 & 0.178 & 0.178 & -0.167 & 8.644 & 0.000 \\
\hline Indiferencia & 2.42 & 2.562 & 0.198 & 0.198 & -0.172 & 9.613 & 0.000 \\
\hline Abuso & 3.83 & 2.199 & 0.101 & 0.101 & -0.082 & 4.895 & 0.000 \\
\hline Autocontrol & 9.75 & 5.997 & 0.135 & 0.135 & -0.073 & 6.551 & 0.000 \\
\hline Madre & 2.86 & 2.895 & 0.188 & 0.188 & -0.161 & 9.155 & 0.000 \\
\hline Indiferencia & 2.31 & 2.398 & 0.194 & 0.194 & -0.167 & 9.468 & 0.000 \\
\hline Abuso & 4.57 & 2.260 & 0.127 & 0.127 & -0.069 & 6.177 & 0.000 \\
\hline Autocontrol & 27.04 & 18.549 & 0.088 & 0.088 & -0.072 & 4.287 & 0.000 \\
\hline $\begin{array}{l}\text { Test de Adicción } \\
\text { al Internet (IAT) }\end{array}$ & 27.04 & 18.549 & 0.088 & 0.088 & -0.072 & 4.287 & 0.000 \\
\hline Tolerancia & 40.01 & 11.420 & 0.096 & 0.095 & -0.096 & 4.661 & 0.000 \\
\hline Uso Excesivo & 13.67 & 4.397 & 0.106 & 0.075 & -0.106 & 5.138 & 0.000 \\
\hline Retirada & 7.20 & 2.263 & 0.184 & 0.108 & -0.184 & 8.968 & 0.000 \\
\hline $\begin{array}{l}\text { Consecuencias } \\
\text { Negativas }\end{array}$ & 7.09 & 2.451 & 0.171 & 0.118 & -0.171 & 8.349 & 0.000 \\
\hline
\end{tabular}

\section{Análisis comparativo}

Se tiene en la Tabla 8 que existen diferencias entre estilos parentales del padre según ubicación geográfica (costa sierra y selva). De igual modo, se encontró diferencias entre los estilos parentales de la madre según ubicación geográfica (costa sierra y selva). De otro lado, se obtuvo diferencias significativas en la adicción al internet y sus dimensiones según ubicación geográfica (costa sierra y selva) 
Maria Matalinares C., Gloria Diaz A., Ornella Raymundo V., Deyvi Baca R., Erika Fernández A.

Tabla 8. Prueba de Kruskal-Wallis de muestras independientes para estimar si existen diferencias de las variables Estilos Parentales y Adicción al Internet según ubicación geográfica

\begin{tabular}{|c|c|c|c|c|c|c|}
\hline Variable & Grupo & $\mathrm{N}$ & $\begin{array}{c}\text { Rango } \\
\text { promedio }\end{array}$ & Chi-cuardrado & $\mathrm{gl}$ & Sig. asintót. \\
\hline \multirow{3}{*}{$\begin{array}{l}\text { Estilos parentales } \\
\text { del padre }\end{array}$} & Costa & 662 & 1077.24 & \multirow{3}{*}{40.976} & \multirow{3}{*}{2} & \multirow{3}{*}{$0.000^{* *}$} \\
\hline & Sierra & 1216 & 1271.44 & & & \\
\hline & Selva & 492 & 1116.21 & & & \\
\hline \multirow{3}{*}{ Indiferencia } & Costa & 662 & 1090.15 & \multirow{3}{*}{29.067} & \multirow{3}{*}{2} & \multirow{3}{*}{$0.000^{* *}$} \\
\hline & Sierra & 1216 & 1256.81 & & & \\
\hline & Selva & 492 & 1137.54 & & & \\
\hline \multirow{3}{*}{ Abuso } & Costa & 662 & 1080.47 & \multirow{3}{*}{58.061} & \multirow{3}{*}{2} & \multirow{3}{*}{$0.000^{* *}$} \\
\hline & Sierra & 1216 & 1287.55 & & & \\
\hline & Selva & 492 & 1071.97 & & & \\
\hline \multirow{3}{*}{ Sobreprotección } & Costa & 662 & 1105.97 & \multirow{3}{*}{25.317} & \multirow{3}{*}{2} & \multirow{3}{*}{$0.000^{* *}$} \\
\hline & Sierra & 1216 & 1253.42 & & & \\
\hline & Selva & 492 & 1124.64 & & & \\
\hline \multirow{3}{*}{$\begin{array}{l}\text { Estilos parentales } \\
\text { de la madre }\end{array}$} & Costa & 662 & 1095.36 & \multirow{3}{*}{35.259} & \multirow{3}{*}{2} & \multirow{3}{*}{$0.000^{* *}$} \\
\hline & Sierra & 1216 & 1266.55 & & & \\
\hline & Selva & 492 & 1106.46 & & & \\
\hline \multirow{3}{*}{ Indiferencia } & Costa & 662 & 1039.72 & \multirow{3}{*}{57.706} & \multirow{3}{*}{2} & \multirow{3}{*}{$0.000^{* *}$} \\
\hline & Sierra & 1216 & 1282.27 & & & \\
\hline & Selva & 492 & 1142.49 & & & \\
\hline \multirow{3}{*}{ Abuso } & Costa & 662 & 1150.88 & \multirow{3}{*}{19.933} & & \\
\hline & Sierra & 1216 & 1242.18 & & 2 & $0.000^{* *}$ \\
\hline & Selva & 492 & 1091.99 & & & \\
\hline & Costa & 662 & 1182.65 & & & \\
\hline Sobreprotección & Sierra & 1216 & 1217.38 & 8.711 & 2 & $0.013^{*}$ \\
\hline & Selva & 492 & 1110.54 & & & \\
\hline & Costa & 662 & 1279.23 & & & \\
\hline Adicción al Internet & Sierra & 1216 & 1205.53 & 45.883 & 2 & $0.000^{* *}$ \\
\hline & Selva & 492 & 1009.88 & & & \\
\hline & Costa & 662 & 1098.76 & & & \\
\hline Tolerancia & Sierra & 1216 & 1165.87 & 40.366 & 2 & $0.000^{* *}$ \\
\hline & Selva & 492 & 1350.74 & & & \\
\hline & Costa & 662 & 1118.54 & & & \\
\hline Uso Excesivo & Sierra & 1216 & 1175.95 & 20.269 & 2 & $0.000^{\star *}$ \\
\hline & Selva & 492 & 1299.22 & & & \\
\hline & Costa & 662 & 1050.26 & & & \\
\hline Retirada & Sierra & 1216 & 1172.74 & 76.059 & 2 & $0.000^{* *}$ \\
\hline & Selva & 492 & 1399.02 & & & \\
\hline & Costa & 662 & 1110.93 & & & \\
\hline $\begin{array}{l}\text { Consecuencias } \\
\text { Neqativas }\end{array}$ & Sierra & 1216 & 1158.20 & 40.199 & 2 & $0.000^{* *}$ \\
\hline & Selva & 492 & 1353.30 & & & \\
\hline
\end{tabular}

$* \mathrm{p}<0.05, * * \mathrm{p}<0.01$ 
Se observa en la Tabla 9 que existen diferencias entre estilos parentales del padre según edad, con excepción del estilo parental sobreprotección $(p>0.05)$. Asimismo, existen diferencias entre los estilos parentales de la madre según edad. En relación a la variable adicción al Internet no se hallo diferencias significativas según edad, al igual que en sus dimensiones tolerancia y uso excesivo; sin embargo, se obtuvieron diferencias significativas en las dimensiones retirada y consecuencias negativas según edad.

Tabla 9. Prueba de Kruskal-Wallis de muestras independientes para estimar si existen diferencias de las variables Estilos Parentales y Adicción al Internet según edad

\begin{tabular}{|c|c|c|c|c|c|c|}
\hline Variable & $\begin{array}{l}\text { Grupo de edad } \\
\text { (años) }\end{array}$ & $\mathrm{N}$ & $\begin{array}{l}\text { Rango } \\
\text { promedio }\end{array}$ & Chi-cuardrado & $\mathrm{gl}$ & Sig. asintót. \\
\hline \multirow{4}{*}{$\begin{array}{l}\text { Estilos parentales } \\
\text { del padre }\end{array}$} & $13-14$ & 813 & 1139.94 & \multirow{4}{*}{10.770} & \multirow{4}{*}{2} & \multirow{4}{*}{$0.005^{* *}$} \\
\hline & $15-16$ & 1339 & 1192.30 & & & \\
\hline & $17-18$ & 218 & 1308.02 & & & \\
\hline & $13-14$ & 813 & 1150.94 & & & \\
\hline \multirow[t]{3}{*}{ Indiferencia } & $15-16$ & 1339 & 1183.61 & \multirow{3}{*}{11.510} & \multirow[t]{3}{*}{2} & \multirow{3}{*}{$0.003^{* *}$} \\
\hline & $17-18$ & 218 & 1326.01 & & & \\
\hline & $13-14$ & 813 & 1124.27 & & & \\
\hline \multirow[t]{3}{*}{ Abuso } & $15-16$ & 1339 & 1202.64 & \multirow[t]{3}{*}{14.222} & \multirow[t]{3}{*}{2} & \multirow[t]{3}{*}{$0.001^{* *}$} \\
\hline & $17-18$ & 218 & 1302.88 & & & \\
\hline & $13-14$ & 813 & 1148.41 & & & \\
\hline \multirow{2}{*}{ Sobreprotección } & $15-16$ & 1339 & 1207.21 & \multirow[t]{2}{*}{3.825} & \multirow[t]{2}{*}{2} & \multirow[t]{2}{*}{0.148} \\
\hline & $17-18$ & 218 & 1190.46 & & & \\
\hline \multirow{4}{*}{$\begin{array}{l}\text { Estilos parentales } \\
\text { de la madre }\end{array}$} & $13-14$ & 813 & 1111.97 & \multirow{4}{*}{17.006} & \multirow{4}{*}{2} & \multirow{4}{*}{$0.000^{*}$} \\
\hline & $15-16$ & 1339 & 1212.51 & & & \\
\hline & $17-18$ & 218 & 1293.81 & & & \\
\hline & $13-14$ & 813 & 1129.59 & & & \\
\hline \multirow[t]{3}{*}{ Indiferencia } & $15-16$ & 1339 & 1190.62 & \multirow[t]{3}{*}{20.598} & \multirow[t]{3}{*}{2} & \multirow[t]{3}{*}{$0.000^{\star *}$} \\
\hline & $17-18$ & 218 & 1362.57 & & & \\
\hline & $13-14$ & 813 & 1117.90 & & & \\
\hline \multirow[t]{3}{*}{ Abuso } & $15-16$ & 1339 & 1206.96 & \multirow[t]{3}{*}{16.574} & 2 & $0.000^{* *}$ \\
\hline & $17-18$ & 218 & 1305.77 & & & \\
\hline & $13-14$ & 813 & 1133.51 & & & \\
\hline Sobreprotección & $15-16$ & 1339 & 1225.21 & 10.554 & 2 & $0.005^{\star *}$ \\
\hline & $17-18$ & 218 & 1135.51 & & & \\
\hline & $13-14$ & 813 & 1172.51 & & & \\
\hline Adicción & $15-16$ & 1339 & 1176.21 & 5.721 & 2 & 0.057 \\
\hline Internet & $17-18$ & 218 & 1290.98 & & & \\
\hline & $13-14$ & 813 & 1190.17 & & & \\
\hline Tolerancia & $15-16$ & 1339 & 1198.06 & 4.658 & 2 & 0.097 \\
\hline & $17-18$ & 218 & 1090.92 & & & \\
\hline & $13-14$ & 813 & 1198.85 & & & \\
\hline Uso Excesivo & $15-16$ & 1339 & 1186.85 & 1.893 & 2 & 0.388 \\
\hline & $17-18$ & 218 & 1127.45 & & & \\
\hline & $13-14$ & 813 & 1220.95 & & & \\
\hline Retirada & $15-16$ & 1339 & 1185.12 & 10.293 & 2 & $0.006^{* *}$ \\
\hline & $17-18$ & 218 & 1055.60 & & & \\
\hline & $13-14$ & 813 & 1211.65 & & & \\
\hline consecuencias & $15-16$ & 1339 & 1187.41 & 6.895 & 2 & $0.032^{*}$ \\
\hline Negativas & $17-18$ & 218 & 1076.24 & & & \\
\hline
\end{tabular}

$* \mathrm{p}<0.05, * * \mathrm{p}<0.01$ 
Según sexo, en la Tabla 10, se hallo diferencias entre estilos parentales del padre de tipo abuso. Asimismo, no se encontró diferencias significativas entre los estilos parentales de la madre según sexo. Sin embargo, se encontró diferencias significativas en la adicción al internet y sus dimensiones según sexo.

Tabla 10. Prueba de U de Mann-Whitney para estimar si existen diferencias de las variables Estilos Parentales y Adicción al Internet según sexo

\begin{tabular}{|c|c|c|c|c|c|c|}
\hline Variable & Grupo & $\mathrm{N}$ & $\begin{array}{c}\text { Rango } \\
\text { promedio }\end{array}$ & $\begin{array}{l}\text { U de Mann- } \\
\text { Whitney }\end{array}$ & Z & Sig. asintót. \\
\hline \multirow{2}{*}{$\begin{array}{l}\text { Estilos parentales del } \\
\text { padre }\end{array}$} & Masculino & 1127 & 1193.78 & \multirow{2}{*}{689,920} & \multirow{2}{*}{-0.596} & \multirow{2}{*}{0.551} \\
\hline & Femenino & 1243 & 1177.04 & & & \\
\hline \multirow{2}{*}{ Indiferencia } & Masculino & 1127 & 1193.12 & \multirow{2}{*}{691,847} & \multirow{2}{*}{-0.521} & \multirow{2}{*}{0.602} \\
\hline & Femenino & 1243 & 1178.59 & & & \\
\hline \multirow{2}{*}{ Abuso } & Masculino & 1127 & 1217.81 & \multirow{2}{*}{662,861} & \multirow{2}{*}{-2.259} & \multirow{2}{*}{$0.024^{*}$} \\
\hline & Femenino & 1243 & 1155.28 & & & \\
\hline \multirow{2}{*}{ Sobreprotección } & Masculino & 1127 & 1192.27 & \multirow{2}{*}{692,805} & \multirow{2}{*}{-0.463} & \multirow{2}{*}{0.643} \\
\hline & Femenino & 1243 & 1179.36 & & & \\
\hline \multirow{2}{*}{$\begin{array}{l}\text { Estilos parentales de } \\
\text { la madre }\end{array}$} & Masculino & 1127 & 1159.19 & \multirow{2}{*}{670,785} & \multirow{2}{*}{-1.786} & \multirow{2}{*}{0.074} \\
\hline & Femenino & 1243 & 1209.35 & & & \\
\hline \multirow{2}{*}{ Indiferencia } & Masculino & 1127 & 1167.88 & \multirow{2}{*}{680,568} & \multirow{2}{*}{-1.209} & \multirow{2}{*}{0.227} \\
\hline & Femenino & 1243 & 1201.48 & & & \\
\hline \multirow{2}{*}{ Abuso } & Masculino & 1127 & 1171.76 & \multirow{2}{*}{684,946} & \multirow{2}{*}{-0.948} & \multirow{2}{*}{0.343} \\
\hline & Femenino & 1243 & 1197.96 & & & \\
\hline \multirow{2}{*}{ Sobreprotección } & Masculino & 1127 & 1159.15 & \multirow{2}{*}{670,730} & \multirow{2}{*}{-1.801} & \multirow{2}{*}{0.072} \\
\hline & Femenino & 1243 & 1209.39 & & & \\
\hline Adingín ototono & Masculino & 1127 & 1271.84 & & & \\
\hline 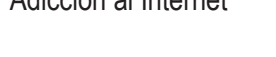 & Femenino & 1243 & 1107.21 & $000,1<2$ & -0.050 & 0.000 \\
\hline & Masculino & 1127 & 1113.47 & & & \\
\hline Iolerancla & Femenino & 1243 & 1250.81 & b19,249 & -4.882 & $0.000^{n}$ \\
\hline Ho & Masculino & 1127 & 1098.03 & 60105 & 11 & 000 \\
\hline Uso EXcesIVo & Femenino & 1243 & 1264.81 & 601,852 & -5.941 & 0.000 \\
\hline Retirada & Masculino & 1127 & 1109.81 & 615121 & & \\
\hline Netilaud & Femenino & 1243 & 1254.12 & (10) & -0.192 & 0.000 \\
\hline Consecuencias & Masculino & 1127 & 1088.35 & $590-20+2$ & 10 & $00000 * *$ \\
\hline Negativas & Femenino & 1243 & 1273.58 & 300,044 & -0.045 & 0.000 \\
\hline
\end{tabular}

$* \mathrm{p}<0.05, * * \mathrm{p}<0.01$ 
De acuerdo a la Tabla 11, se halló diferencias entre estilos parentales del padre de tipo abuso y sobreprotección según nivel de instrucción. De igual modo, se detectó diferencias significativas entre los estilos parentales de la madre de tipo abuso y sobreprotección según nivel de instrucción. Sin embargo, no existen diferencias significativas en la adicción al Internet y sus dimensiones según nivel de instrucción.

Tabla 11. Prueba de Kruskal-Wallis de muestras independientes para estimar si existen diferencias de las variables Estilos Parentales y Adicción al Internet según nivel de instrucción

\begin{tabular}{|c|c|c|c|c|c|c|}
\hline Variable & Grupo & N & $\begin{array}{c}\text { Rango } \\
\text { promedio }\end{array}$ & Chi-cuardrado & $\mathrm{gl}$ & Sig. asintót \\
\hline \multirow{4}{*}{$\begin{array}{l}\text { Estilos parentales } \\
\text { del padre }\end{array}$} & Tercero & 759 & 1131.71 & & & \\
\hline & Cuarto & 811 & 1231.52 & 8.404 & 2 & $0.015^{\star}$ \\
\hline & Quinto & 800 & 1188.33 & & & \\
\hline & Tercero & 759 & 1151.83 & & & \\
\hline \multirow[t]{3}{*}{ Indiferencia } & Cuarto & 811 & 1209.19 & 2.977 & 2 & 0.226 \\
\hline & Quinto & 800 & 1193.43 & & & \\
\hline & Tercero & 759 & 1102.74 & & & \\
\hline \multirow[t]{3}{*}{ Abuso } & Cuarto & 811 & 1245.17 & 18.316 & 2 & $0.000^{* *}$ \\
\hline & Quinto & 800 & 1201.94 & & & \\
\hline & Tercero & 759 & 1137.49 & & & \\
\hline \multirow[t]{2}{*}{ Sobreprotección } & Cuarto & 811 & 1241.57 & 9.590 & 2 & $0.008^{* *}$ \\
\hline & Quinto & 800 & 1174.21 & & & \\
\hline \multirow{4}{*}{$\begin{array}{l}\text { Estilos parentales } \\
\text { de la madre }\end{array}$} & Tercero & 759 & 1110.65 & & & \\
\hline & Cuarto & 811 & 1205.90 & 14.192 & 2 & $0.001^{* *}$ \\
\hline & Quinto & 800 & 1235.83 & & & \\
\hline & Tercero & 759 & 1140.36 & & & \\
\hline \multirow[t]{3}{*}{ Indiferencia } & Cuarto & 811 & 1190.74 & 5.897 & 2 & 0.052 \\
\hline & Quinto & 800 & 1223.01 & & & \\
\hline & Tercero & 759 & 1115.29 & & & \\
\hline \multirow[t]{3}{*}{ Abuso } & Cuarto & 811 & 1213.51 & 12.279 & 2 & $0.002^{* *}$ \\
\hline & Quinto & 800 & 1223.71 & & & \\
\hline & Tercero & 759 & 1093.76 & & & \\
\hline \multirow[t]{3}{*}{ Sobreprotección } & Cuarto & 811 & 1220.02 & 20.700 & 2 & $0.000^{* *}$ \\
\hline & Quinto & 800 & 1237.54 & & & \\
\hline & Tercero & 759 & 1177.22 & & & \\
\hline \multirow[t]{3}{*}{ Adicción al Internet } & Cuarto & 811 & 1188.48 & 0.167 & 2 & 0.920 \\
\hline & Quinto & 800 & 1190.34 & & & \\
\hline & Tercero & 759 & 1189.08 & & & \\
\hline \multirow[t]{3}{*}{ Tolerancia } & Cuarto & 811 & 1180.08 & 0.079 & 2 & 0.961 \\
\hline & Quinto & 800 & 1187.59 & & & \\
\hline & Tercero & 759 & 1198.09 & & & \\
\hline \multirow[t]{3}{*}{ Uso Excesivo } & Cuarto & 811 & 1188.81 & 0.679 & 2 & 0.712 \\
\hline & Quinto & 800 & 1170.20 & & & \\
\hline & Tercero & 759 & 1223.40 & & & \\
\hline \multirow[t]{2}{*}{ Retirada } & Cuarto & 811 & 1168.71 & 3.516 & 2 & 0.172 \\
\hline & Quinto & 800 & 1166.57 & & & \\
\hline \multirow{3}{*}{$\begin{array}{l}\text { Consecuencias } \\
\text { Negativas }\end{array}$} & Tercero & 759 & 1191.02 & & & \\
\hline & Cuarto & 811 & 1211.54 & 2.994 & 2 & 0.224 \\
\hline & Quinto & 800 & 1153.87 & & & \\
\hline
\end{tabular}

$* \mathrm{p}<0.05, * * \mathrm{p}<0.01$ 
Se aprecia en la Tabla 12, que hay diferencias entre estilos parentales del padre de tipo abuso y sobreprotección según tipo de apoderado(s). Asimismo, se detectó diferencias significativas en todos los estilos parentales de la madre con respecto al tipo de apoderado(s). En contraste, no se hallo diferencias significativas en la adicción al internet y sus dimensiones según tipo de apoderado(s).

Tabla 12. Prueba de Kruskal-Wallis de muestras independientes para estimar si existen diferencias de las variables Estilos Parentales y Adicción al Internet según tipo de apoderado(s)

\begin{tabular}{|c|c|c|c|c|c|c|}
\hline Variable & Grupo & $\mathrm{N}$ & $\begin{array}{c}\text { Rango } \\
\text { promedio }\end{array}$ & Chi-cuadrado & $\mathrm{gl}$ & Sig. asintót. \\
\hline \multirow{4}{*}{$\begin{array}{l}\text { Estilos parentales } \\
\text { del padre }\end{array}$} & Padre y madre & 1611 & 1210.38 & \multirow{4}{*}{10.723} & \multirow{4}{*}{3} & \multirow{4}{*}{$0.013^{*}$} \\
\hline & Sólo padre & 141 & 1214.34 & & & \\
\hline & Sólo madre & 435 & 1092.63 & & & \\
\hline & Otro apoderado & 183 & 1158.66 & & & \\
\hline \multirow{4}{*}{ Indiferencia } & Padre y madre & 1611 & 1187.16 & \multirow{4}{*}{1.068} & \multirow{4}{*}{3} & \multirow{4}{*}{0.785} \\
\hline & Sólo padre & 141 & 1183.07 & & & \\
\hline & Sólo madre & 435 & 1163.72 & & & \\
\hline & Otro apoderado & 183 & 1224.51 & & & \\
\hline \multirow{4}{*}{ Abuso } & Padre y madre & 1611 & 1212.00 & \multirow{4}{*}{10.280} & \multirow{4}{*}{3} & \multirow{4}{*}{$0.016^{*}$} \\
\hline & Sólo padre & 141 & 1200.61 & & & \\
\hline & Sólo madre & 435 & 1104.06 & & & \\
\hline & Otro apoderado & 183 & 1127.84 & & & \\
\hline \multirow{4}{*}{ Sobreprotección } & Padre y madre & 1611 & 1249.05 & \multirow{4}{*}{66.089} & \multirow{4}{*}{3} & \multirow{4}{*}{$0.000^{* *}$} \\
\hline & Sólo padre & 141 & 1269.39 & & & \\
\hline & Sólo madre & 435 & 966.39 & & & \\
\hline & Otro apoderado & 183 & 1082.27 & & & \\
\hline \multirow{4}{*}{$\begin{array}{l}\text { Estilos parentales } \\
\text { de la madre }\end{array}$} & Padre y madre & 1611 & 1167.88 & \multirow{4}{*}{14.234} & \multirow{4}{*}{3} & \multirow{4}{*}{$0.003^{* *}$} \\
\hline & Sólo padre & 141 & 1069.49 & & & \\
\hline & Sólo madre & 435 & 1283.98 & & & \\
\hline & Otro apoderado & 183 & 1195.91 & & & \\
\hline \multirow{4}{*}{ Indiferencia } & Padre y madre & 1611 & 1167.12 & \multirow{4}{*}{8.095} & \multirow{4}{*}{3} & \multirow{4}{*}{$0.044^{*}$} \\
\hline & Sólo padre & 141 & 1121.88 & & & \\
\hline & Sólo madre & 435 & 1258.84 & & & \\
\hline & Otro apoderado & 183 & 1221.98 & & & \\
\hline
\end{tabular}


Influencia de los estilos parentales en la adicción al Internet en alumnos de secundaria del Perú

\begin{tabular}{|c|c|c|c|c|c|c|}
\hline \multirow{4}{*}{ Abuso } & Padre y madre & 1611 & 1158.68 & \multirow{4}{*}{12.889} & \multirow{4}{*}{3} & \multirow{4}{*}{$0.005^{\star *}$} \\
\hline & Solo padre & 141 & 1138.86 & & & \\
\hline & Solo madre & 435 & 1281.41 & & & \\
\hline & Otro apoderado & 183 & 1229.53 & & & \\
\hline \multirow{5}{*}{ Sobreprotección } & Padre y madre & 1611 & 1183.49 & \multirow{5}{*}{13.679} & \multirow{5}{*}{3} & \multirow{5}{*}{$0.003^{* *}$} \\
\hline & Solo padre & 141 & 1025.60 & & & \\
\hline & Solo madre & 435 & 1260.27 & & & \\
\hline & Otro apoderado & 183 & 1148.71 & & & \\
\hline & Padre y madre & 1611 & 1191.09 & & & \\
\hline \multirow{3}{*}{$\begin{array}{l}\text { Adicción al } \\
\text { Internet }\end{array}$} & Solo padre & 141 & 1059.84 & \multirow{3}{*}{5.441} & \multirow{3}{*}{3} & \multirow{3}{*}{0.142} \\
\hline & Solo madre & 435 & 1209.61 & & & \\
\hline & Otro apoderado & 183 & 1175.82 & & & \\
\hline \multirow{4}{*}{ Tolerancia } & Padre y madre & 1611 & 1182.68 & \multirow{4}{*}{3.960} & \multirow{4}{*}{3} & \multirow{4}{*}{0.266} \\
\hline & Solo padre & 141 & 1288.64 & & & \\
\hline & Solo madre & 435 & 1158.31 & & & \\
\hline & Otro apoderado & 183 & 1195.51 & & & \\
\hline \multirow{4}{*}{ Uso Excesivo } & Padre y madre & 1611 & 1177.30 & \multirow{4}{*}{7.230} & \multirow{4}{*}{3} & \multirow{4}{*}{0.065} \\
\hline & Sólo padre & 141 & 1332.60 & & & \\
\hline & Sólo madre & 435 & 1164.40 & & & \\
\hline & Otro apoderado & 183 & 1194.51 & & & \\
\hline \multirow{4}{*}{ Retirada } & Padre y madre & 1611 & 1173.61 & \multirow{4}{*}{3.020} & \multirow{4}{*}{3} & \multirow{4}{*}{0.389} \\
\hline & Sólo padre & 141 & 1272.80 & & & \\
\hline & Sólo madre & 435 & 1195.98 & & & \\
\hline & Otro apoderado & 183 & 1198.03 & & & \\
\hline \multirow{4}{*}{$\begin{array}{l}\text { Consecuencias } \\
\text { Negativas }\end{array}$} & Padre y madre & 1611 & 1179.62 & \multirow{4}{*}{3.223} & \multirow{4}{*}{3} & \multirow{4}{*}{0.358} \\
\hline & Sólo padre & 141 & 1284.72 & & & \\
\hline & Sólo madre & 435 & 1177.10 & & & \\
\hline & Otro apoderado & 183 & 1180.79 & & & \\
\hline
\end{tabular}

$* \mathrm{p}<0.05, * * \mathrm{p}<0.01$

\section{Análisis de causalidad}

Como se observa en la Tabla 13 se aplicó la prueba chi-cuadrado entre el estilo parental de indiferencia del padre y la adicción al Internet, encontrándose depen- 
dencia entre ambas variables $(\mathrm{p}<0.01)$. Y, el efecto del estilo parental de indiferencia del padre sobre la adicción al Internet fue de 0.162 , nivel considerado bajo (Clark-Carter, 2002).

Tabla 13. Prueba chi-cuadrado y W de Cohen entre el estilo parental de indiferencia del padre y la adicción al Internet en el adolescente escolar

\begin{tabular}{lccc}
\hline & Valor & gl & Sig. asintótica (bilateral) \\
\hline Chi-cuadrado de Pearson & $62.559^{\mathrm{a}}$ & 4 & 0.000 \\
Coeficiente de contingencia & 0.160 & & 0.000 \\
W de Cohen & 0.162 & & \\
$\mathrm{~N}$ de casos válidos & 2370 & & \\
\hline
\end{tabular}

a. 0 casillas $(0,0 \%)$ tienen una frecuencia esperada inferior a 5. La frecuencia mínima esperada es 47.62.

Se obtuvo en la Tabla 14 una relación de dependencia $(\mathrm{p}<0.01)$ entre el estilo parental de abuso del padre y la adicción al Internet a través de la prueba chicuadrado. Y, el efecto del estilo parental de abuso del padre sobre la adicción al Internet fue de 0.184 , nivel considerado bajo (Clark-Carter, 2002).

Tabla 14. Prueba chi-cuadrado y W de Cohen entre el estilo parental de abuso del padre y la adicción al Internet en el adolescente escolar

\begin{tabular}{lccc}
\hline & Valor & gl & Sig. asintótica (bilateral) \\
\hline Chi-cuadrado de Pearson & $79.868^{\mathrm{a}}$ & 4 & 0.000 \\
Coeficiente de contingencia & 0.181 & & 0.000 \\
W de Cohen & 0.184 & & \\
$\mathrm{~N}$ de casos válidos & 2369 & & \\
\hline
\end{tabular}

a. 0 casillas $(0,0 \%)$ tienen una frecuencia esperada inferior a 5. La frecuencia mínima esperada es 45.28.

De acuerdo a la Tabla 15 se aplicó la prueba chi-cuadrado entre el estilo parental de sobreprotección del padre y la adicción al Internet, en donde se halló una relación de dependencia entre ambas variables $(\mathrm{p}<0.01)$. Y, el efecto del estilo parental de sobreprotección del padre sobre la adicción al Internet fue de 0.134, nivel considerado bajo (Clark-Carter, 2002).

Tabla 15. Prueba chi-cuadrado y W de Cohen entre el estilo parental de sobreprotección del padre y la adicción al Internet en el adolescente escolar

\begin{tabular}{lccc}
\hline & Valor & gl & Sig. asintótica (bilateral) \\
\hline Chi-cuadrado de Pearson & $42.856^{\mathrm{a}}$ & 8 & 0.000 \\
Coeficiente de contingencia & 0.133 & & 0.000 \\
W de Cohen & 0.134 & & \\
\hline N de casos válidos & 2370 & & \\
\hline
\end{tabular}

a. 0 casillas $(0,0 \%)$ tienen una frecuencia esperada inferior a 5 . La frecuencia mínima esperada es 26.86. 
En la Tabla 16 se aplicó la prueba chi-cuadrado entre el estilo parental de indiferencia de la madre y la adicción al Internet, encontrándose una relación de dependencia entre ambas variables $(\mathrm{p}<0.01)$. Y, el efecto del estilo parental de indiferencia de la madre sobre la adicción al Internet fue de 0.141 , nivel considerado bajo (Clark-Carter, 2002).

Tabla 16. Prueba chi-cuadrado y $\mathrm{W}$ de Cohen entre el estilo parental de indiferencia de la madre y la adicción al Internet en el adolescente escolar

\begin{tabular}{lccc}
\hline & Valor & gl & Sig. asintótica (bilateral) \\
\hline Chi-cuadrado de Pearson & $47.018^{\mathrm{a}}$ & 4 & 0.000 \\
Coeficiente de contingencia & 0.139 & & 0.000 \\
W de Cohen & 0.141 & & \\
\hline $\mathrm{N}$ de casos válidos & 2370 & & \\
\hline
\end{tabular}

a. 0 casillas $(0,0 \%)$ tienen una frecuencia esperada inferior a 5. La frecuencia mínima esperada es 48.09.

Se observa en la Tabla 17 que el estilo parental de abuso de la madre y la adicción al Internet tuvieron una relación de dependencia $(\mathrm{p}<0.01)$ a través de la prueba chi-cuadrado. Siendo el efecto del estilo parental de indiferencia de la madre sobre la adicción al Internet fue de 0.158, nivel considerado bajo (Clark-Carter, 2002).

Tabla 17. Prueba chi-cuadrado y W de Cohen entre el estilo parental de abuso de la madre y la adicción al Internet en el adolescente escolar

\begin{tabular}{lccc}
\hline & Valor & gl & Sig. asintótica (bilateral) \\
\hline Chi-cuadrado de Pearson & $58.966^{\mathrm{a}}$ & 4 & 0.000 \\
Coeficiente de contingencia & 0.156 & & 0.000 \\
$W$ de Cohen & 0.158 & & \\
\hline $\mathrm{N}$ de casos válidos & 2370 & & \\
\hline
\end{tabular}

a. 0 casillas $(0,0 \%)$ tienen una frecuencia esperada inferior a 5 . La frecuencia mínima esperada es 40.70 .

Se aprecia en la Tabla 18 que el estilo parental de sobreprotección de la madre y la adicción al Internet presentan una relación de dependencia $(\mathrm{p}<0.01)$. De otro lado, el efecto del estilo parental de sobreprotección de la madre sobre la adicción al Internet fue de 0.150, nivel considerado bajo (Clark-Carter, 2002).

Tabla 18. Prueba chi-cuadrado y W de Cohen entre el estilo parental de sobreprotección de la madre y la adicción al Internet en el adolescente escolar

\begin{tabular}{lccc}
\hline & Valor & gl & Sig. asintótica (bilateral) \\
\hline Chi-cuadrado de Pearson & $53.412^{\mathrm{a}}$ & 4 & 0.000 \\
Coeficiente de contingencia & 0.148 & & 0.000 \\
W de Cohen & 0.150 & & \\
$\mathrm{~N}$ de casos válidos & 2370 & & \\
\hline
\end{tabular}

a. 0 casillas $(0,0 \%)$ tienen una frecuencia esperada inferior a 5 . La frecuencia mínima esperada es 51.14. 


\section{DISCUSIÓN DE RESULTADOS}

El presente estudio fue realizado a nivel nacional con estudiantes de $3 .^{\text {ro }}$ a $5 .^{\text {to }}$ año de secundaria de 14 ciudades representativas del Perú, con edades entre los 13 y 18 años. Las características encontradas en los alumnos son las siguientes: El $53.8 \%$ de los adolescentes y $60.3 \%$ de las adolescentes permanecen al menos 01 hora diaria en Internet; seguido del uso entre 01 a 05 horas diarias en varones y mujeres representado por el $40.6 \%$ y $35.4 \%$, respectivamente (Véase tabla 2). Estos datos muestran un incremento en comparación a las cifras reportadas el 2012 por IPSOS Apoyo, quienes informaron que el $25 \%$ de los adolescentes que viven en Lima Metropolitana ingresan todos los días al Internet, incrementándose la cifra en los adolescentes de 17 a 20 años en un 38\%. Si bien el presente estudio extrajo datos a nivel nacional, se aprecia un incremento en el uso del Internet en los adolescentes, los mismos que pueden relacionarse a la facilidad en la accesibilidad al Internet en diferentes partes del país ya sea por cabinas o en casa. Cabe resaltar, que en solo un año se aprecia un incremento significativo, en tal sentido el adolescente peruano se encuentra en un contexto de vulnerabilidad para el uso excesivo del Internet (adicción al Internet).

De acuerdo al contenido accedido por Internet se encuentra en los varones en mayor medida las redes sociales $(21.8 \%)$, seguido por redes sociales y actividades escolares (19\%), y juegos online, redes sociales y contenido sexual (19\%). En contraste, la mujeres accedieron a redes sociales y actividades escolares (35.6\%), seguido de actividades escolares (25.5\%) (Véase tabla 3). Datos que reflejan los hallazgos de IPSOS Apoyo del 2012, quienes reportan que el $78 \%$ de adolescentes de 13 a 16 años y el 84\% de adolescentes de 17 a 20 años tienen cuenta el Facebook con un uso frecuente, en tal sentido se aprecia que el adolescente peruano tiene un mayor acceso de las redes sociales al paralelo de la ejecución de sus actividades escolares, dichas redes sirven como medio de comunicación para la realización de las actividades escolares grupales, sin embargo puede servir como un factor predisponente para el desarrollo de la adicción al Internet considerando lo expuesto por Cruzado, Matos y Kendall (2006), quienes informan que los más jóvenes son más vulnerables a los potenciales efectos negativos del Internet y que por la búsqueda de novedades e impulsividad propias de su edad, los adolescentes son más susceptibles a desarrollar problemas adictivos.

Es importante considerar que si bien los adolescentes se encuentran expuestos a estímulos sociales que pueden promover el uso excesivo del Internet, se debe resaltar que la familia es la primera fuente de socialización del niño y adolescente (Bronfenbrenner, 1979), por lo cual hacemos un análisis descriptivo de los estilos parentales disfuncionales que los adolescentes perciben en su crianza. Se obtuvo que en el estilo parental del padre de tipo indiferencia, entendido como la ausencia de exigencias y de cumplimiento de responsabilidades, falta de estructuración, control y apoyo, es percibido en un nivel alto por el $15.88 \%$ de los adolescentes y el $18.26 \%$ de las adolescentes, cifras consideradas altas. En el estilo parental de abuso, entendido como la imposición de las reglas, esperan una obediencia 
estricta y se basan en el poder que estos tienen como padres, así como se generan castigos físicos y verbales, es percibido en un nivel alto por el $18.73 \%$ y $14.88 \%$ de varones y mujeres, respectivamente. Y, en el estilo parental de sobreprotección, referida al uso de un bajo control y exigencia con poca sensibilidad hacia las necesidades de los hijos, muchas veces pueden ser crueles y no establecen normas, no se muestran firmes frente a sus hijos y no esperan acciones maduras de sus hijos. La comunicación no es efectiva y suele ir en una sola dirección de padre al hijo, este estilo es percibido en un nivel alto por $8.87 \%$ de los adolescentes y $10.38 \%$ de las adolescentes (véase Tabla 4). Las cifras encontradas en un nivel alto son consideradas significativas, ya que reflejan que los estilos parentales disfuncionales tienen una alta incidencia en los adolescentes peruanos, esta estaría relacionada a factores socioculturales, sin embargo es importante resaltar que algunas investigaciones demuestran que una educación democrática, que combina la disciplina moderada con la explicación razonada del motivo de la mala actuación, se asocia con distintos comportamientos que se consideran positivos en diversas áreas del desarrollo tanto en niños como adolescentes (Osorio et al., 2009).

En los estilos parentales de la madre. El estilo parental de indiferencia es percibido en un nivel alto por los adolescentes y las adolescentes en un $16.33 \%$ y $18.18 \%$, respectivamente. Asimismo, se encontró que estilo parental de abuso es percibido en un nivel alto por $15 \%$ y $14.32 \%$ de varones y mujeres, respectivamente. Y, el estilo parental sobreprotección es percibido en un nivel alto por el $19.61 \%$ de los adolescentes y $18.02 \%$ de las adolescentes (véase Tabla 5). A diferencia de la percepción de los estilos parentales del padre, los adolescentes perciben que la figura materna genera mayor sobreprotección, sin embargo los estilos parentales disfuncionales se evidencian también en cifras altas.

En cuanto a los niveles de Adicción al Internet, los varones se ubicaron en un nivel muy alto el $11.27 \%$; así como el $12.39 \%$ de mujeres se halló en dicho nivel (véase tabla 6). Dichas cifras refieren que los adolescentes presentan altos indicadores de tolerancia, uso excesivo, dificultad en la retirada o limitar su uso del Internet y la percepción de consecuencias negativas por el uso, en tal sentido se encuentra en un cuadro de adicción al Internet. Son cifras elevadas y por tanto se discrepa con lo referido por Prince (2011), quien informa que la prevalencia de la adicción a Internet es la más baja entre los adolescentes, con un alcance de 4,6 a $4,7 \%$. Ese número aumenta entre la población general la población de usuarios de Internet, con rangos de 6 a $15 \%$ de la población general presentan los signos de la adicción, y que va hasta el 13 al 18,4\% entre los estudiantes universitarios, que parecen estar en mayor riesgo. Estos números estiman el alcance del problema y sugieren que una proporción significativa de usuarios en línea pueden sufrir uno o más signos de adicción a Internet. Sin embargo se aprecia que conforme pasan los años las necesidades de mantener actividades escolares y académicas al paralelo del uso del Internet están incrementando rápidamente las estadísticas de adicción o uso excesivo. 
¿Realmente serán diferentes los niveles de adicción entre estudiantes educación superior con los de secundaria? A este respecto, es importante, continuar con las investigaciones.

Como se recuerda, el objetivo de esta investigación se orientó a identificar el nivel de influencia de los estilos parentales disfuncionales sobre la adicción al Internet, obteniéndose que los estilos parentales influyen sobre la adicción al Internet en estudiantes de secundaria del Perú, siendo los estilos parentales de indiferencia y abuso del padre los que mejor explican la adicción al Internet (véase Tablas $13,14)$, lo cual implica que los estilos parentales disfuncionales de indiferencia y abuso percibido por los adolescentes en la figura paterna, contribuyen a que el adolescente desarrolle conductas de uso excesivo del Internet. Datos reforzados por diversas investigaciones que demuestran como un estilo educativo parental inadecuado puede ser el precursor de diversas problemáticas durante la adolescencia. Autores como Justicia, Benites, Pichardo, Fernandez, García $(2006 ; 2009)$ citados por Vergara (2009), concluyen en sus investigaciones que los padres con características de comportamiento restrictivo, negligente, con falta de afecto, con hostilidad, disciplina inconsistente y falta de supervisión son el desencadenante de problemas de sus hijos. Así mismo, Hidin (2005) hizo un estudio en jóvenes filipinos, en donde encontró que en los hogares dónde los padres toman decisiones conjuntas y son democráticos, sus hijos tienen alta probabilidad de tener un mejor rendimiento académico y terminar la escuela y los hijos de padres negligentes son los que menos posibilidades tienen de lograr un desempeño académico aceptable. Se aprecia la importancia de los estilos parentales en la predisposición a la adquisición de conductas adictivas, en tal sentido se concuerda con Estévez y Calvete (2007), quien en su estudio sobre estilos parentales y juego patológico concinciden en señalar a los estilos negligente y permisivo como uno de los factores de riesgo para desarrollar este tipo de patología. Ante los resultados expuestos se infiere que una estrategia de intervención preventiva es abordar los estilos parentales desde las primeras edades y generar un nivel de control y disciplina democrática.

En el análisis comparativo se obtuvo que hay diferencias significativas en la adicción al Internet y sus dimensiones según ubicación geográfica (costa sierra y selva), identificándose un cierto predominio en la adicción al Internet en la dimensión de tolerancia en la zona Selva, lo cual indica que los adolescentes de la Selva perciben mayor necesidad de un mejor equipamiento informático, un nuevo programa, o pasar más horas de uso, como una reacción compulsiva para aliviar la ansiedad de no estar conectado, o ansiedad por situaciones estresantes de la vida cotidiana; sin embargo a nivel de uso excesivo se apreció que se da más en la selva, lo que implica que los adolescentes de la selva tienden a perder el sentido del tiempo, ya que no logran controlar la necesidad de estar conectados al Internet, dejando de lado actividades sociales y familiares por pensar y estar conectados en línea, pasando más tiempo del que se había programado; mientras que las dimensiones de retirada y consecuencias negativas tiene un mayor predominio en también en la zona selva, en tal sentido, los adolescentes que viven en la Selva tienden a presentar más 
sentimientos de ira, ansiedad, y/o depresión cuando la computadora es inaccesible, así como emiten argumentos, mentiras, bajo rendimiento, aislamiento social, fatiga y descuido en las actividades domésticas como consecuencia de estar conectado al Internet. Estos resultados concuerdan con los reportes realizados por Estévez y Calvete (2007), quien afirma que las personas con juego patológico puntuaban más alto en diversos comportamientos disfuncionales con predominio en desconexión (asociado a la variable retirada), rechazo (asociado a la variable tolerancia) y autonomía deteriorada (asociado a la variable de consecuencias negativas); por su parte en cuanto a los estilos de crianza durante su adolescencia fue más influyente negativamente el de la figura paterna, encontrando una relación importante con aquellos estilos de crianza autoritarios caracterizados por la impulsividad y en definitiva con los totalmente faltos de disciplina. Dicha afirmación reafirman lo hallado en el presente estudio, identificando que las conductas adictivas guardan relación con estilos de crianza disfuncionales, cabe considerar que el análisis general reporta una mayor incidencia de adolescentes con adicción al Internet en la región costa (véase Tabla 8). Así mismo, los estilos parentales de la madre y del padre difieren según ubicación geográfica (costa sierra y selva), identificándose un mayor predominio de los estilos parentales disfuncionales de la figura materna en la zona sierra del Perú, en base al hallazgo obtenido se puede inferir que los adolescentes de la sierra presentan factores de predisposición para la adicción al Internet, considerando que en dicha zona tienen una mayor prevalencia de estilos de crianza disfuncionales.

Según la edad hay diferencias entre estilos parentales del padre y madre, identificándose que los adolescentes con edades entre 17 y 18 años tienden a percibir con mayor intensidad estilos parentales disfuncionales de abuso e indiferencia por parte de ambos progenitores, mientras que los adolescentes con edades entre $15 \mathrm{y}$ 16 años perciben mayor sobreprotección solo por parte de la figura materna (véase Tabla 9). Es importante considerar que los estilos parentales forman parte de un proceso de interacción, ante ello podemos tener en cuenta que durante esta etapa de la adolescencia, está la relación con los padres, la cual puede sufrir alteraciones o tensiones relacionadas con los dramáticos cambios que experimentan los adolescentes y la influencia que esto tiene en los padres (De la Espriella, 1995).

En la adicción al Internet se obtuvieron diferencias significativas en las dimensiones retirada y consecuencias negativas según edad, evidenciándose un predominio en adolescentes con edades entre 13 y 14 años (véase Tabla 9), lo cual indica que los jóvenes entre estas edades tienden a presentar dificultades con el control y respeto del tiempo de estar conectados, así como presentan mayores consecuencias negativas por estar en línea. Dichos datos guardarían relación al escaso control de impulsos propios de la edad, tal como Rodrigo et, al. (2004) afirman que los importantes cambios biológicos que experimenta el adolescente intervienen en el proceso de desarrollo general y en la búsqueda de armonía y equilibrio consigo mismo y con su integración a la sociedad, y los modelos sociales dentro del entorno son los más influyentes en este proceso. 
Según el sexo se obtuvo diferencias entre estilos parentales del padre de tipo abuso, identificándose que el género masculino tiende a percibir más este estilo parental disfuncional (véase Tabla 10), lo cual denota patrones culturales que estarían relacionados a la crianza del género masculino en el Perú. Cabe resaltar que diversos estudios han probado que los estilos parentales pueden predecir la opinión de los adolescentes sobre los efectos de las practicas parentales que sus padres han tenido con ellos; de esta forma se afirma que se puede esperar que las variables representan los factores relacionados con la exigencia y tienen un efecto más benéfico cuando se dan en una configuración o estilo democrático, que cuando se dan en una autoritaria (Raya, 2008, p. 26).

En cuanto a la adicción al Internet se obtuvo que existen diferencias significativas entre los varones y mujeres, con predominio del género masculino (véase tabla 10), lo cual implica que en los varones se encuentran indicadores de adicción al Internet más significativos, si bien se aprecia una cercanía porcentual en las mujeres, se puede inferir que las varones tienen mayores utilidades del Internet que le generan mayor adicción, juegos en línea, redes sociales, buscar información, entre otros; a diferencia de las mujeres que usan el Internet más para actividades académicas y redes sociales. Es importante considerar que mediante el intercambio de mensajes en línea, los usuarios tratan de compensar lo que les puede faltar en la vida real (Caplan, 2007). Ellos pueden ser capaces de utilizar el chat, la red social, para encontrar el significado psicológico y de conexión, forman rápidamente vínculos íntimos, y se sienten emocionalmente cerca de los demás, unido a ello la necesidad de todo adolescente de pertenecer a un grupo social.

Hay diferencias entre estilos parentales del padre de tipo abuso y sobreprotección según tipo de apoderado(s), indicando que los adolescentes que viven solo con el padre perciben mayor sobreprotección, mientras que los adolescentes que viven con ambos padres perciben mayor abuso. Asimismo, se detectó diferencias significativas en todos los estilos parentales de la madre, indiferencia, abuso y sobreprotección, según el tipo de apoderado(s), identificándose que los adolescentes que viven solo con su madre perciben mayor indiferencia, abuso y sobreprotección por parte de esta (véase Tabla 12). Dichos hallazgos estarían relacionados a los múltiples roles que cumple la mujer en la sociedad, considerando que su condición le genera la necesidad de desenvolverse en diversos roles, lo cual promueve que haya una disminución en el tiempo y calidad del vínculo establecido con sus hijos. Cabe destacar que Musitu (2000) introduce la cultura como un componente que participa en la educación de los hijos, el autor afirma que los rasgos culturales intervienen directamente en los valores de los padres y modifican el estilo de interacción dentro del núcleo familiar. En tal sentido, se resalta la importancia de estudiar los factores sociales asociados a la crianza monoparental, ya que en el presente estudio se ha detectado un porcentaje significativo de estilos parentales disfuncionales en este tipo de familias. 
En síntesis, los hallazgos obtenidos nos muestran un incremento de la incidencia de adicción al Internet en los adolescentes del Perú, en contraste a las cifras halladas en estudios previos, así como se afirma la causalidad de los estilos parentales disfuncionales (indiferencia, abuso y sobreprotección) en la variable adicción al Internet, detectándose que los jóvenes de 13 a 19 años que viven en la zona costa presentan más indicadores de adicción al Internet, y los adolescentes de la sierra perciben estilos parentales más disfuncionales, aunado a ello que los hogares monoparentales donde el cuidado lo ejerce uno de los progenitores, presentan mayor percepción de estilos parentales disfuncionales. Por ende, los adolescentes que presentan dichas características sociofamiliares se encuentran en situación de vulnerabilidad y una intervención temprana y oportuna podría prevenir cuadros de adicción al Internet.

\section{CONCLUSIONES}

1. Los estilos parentales influyen sobre la adicción al Internet en estudiantes de secundaria del Perú, siendo los estilos parentales de indiferencia y abuso del padre los que mejor explican la adicción al Internet.

2. Hay diferencias significativas en la adicción al Internet y sus dimensiones según ubicación geográfica (costa sierra y selva), siendo los adolescentes de la costa los que presentan una mayor adicción al Internet.

3. Los estilos parentales de la madre y del padre difieren según ubicación geográfica (costa sierra y selva), poseyendo un peor estilo parental de la madre y padre en los adolescentes de la sierra.

4. Hay diferencias entre estilos parentales del padre según edad, con excepción del estilo parental sobreprotección; donde los adolescentes entre los 17 y 18 años poseen peores estilos parentales. Asimismo, existen diferencias entre los estilos parentales de la madre según edad; de igual manera, teniendo un peor estilo parental los adolescente entre los 17 y 18 años.

5. En la adicción al Internet la edad no es un factor importante en las dimensiones tolerancia y uso excesivo; sin embargo, sí es importante en las dimensiones retirada y consecuencias negativas, siendo más pronunciado entre los 13 y 14 años.

6. El estilo parental del padre de tipo abuso se mostró diferente según sexo poseyendo un peor nivel los varones. No siendo diferente los estilos de parentales de la madre según sexo.

7. Existen diferencias significativas en la adicción al Internet siendo más pronunciado en los varones.

8. Los estilos parentales del padre de tipo abuso y sobreprotección difieren según nivel de instrucción, siendo más pronunciado en los estudiantes del cuarto de secundaria. De igual modo, se detectó diferencias significativas entre los estilos parentales de la madre de tipo abuso y sobreprotección según nivel de instrucción, siendo más relevante en los estudiantes de quinto de secundaria. 
9. No existen diferencias significativas en la adicción al Internet y sus dimensiones según nivel de instrucción.

10. Hay diferencias entre estilos parentales del padre de tipo abuso y sobreprotección según tipo de apoderado(s), poseyendo peores estilos parentales los que fueron criados solo por su padre. Asimismo, se detectó diferencias significativas en todos los estilos parentales de la madre con respecto al tipo de apoderado(s), siendo más pronunciado en aquellos que fueron criados solo por su madre.

11. En la adicción al Internet y sus dimensiones según tipo de apoderado(s), no se obtuvo diferencias significativas.

\section{REFERENCIAS BIBLIOGRÁFICAS}

Aboujaoude, E. (2010). Uso problemático de Internet: un panorama general. Worl Psychiatry (Ed. Esp.). 8(02), pp. 85-90.

Baumrind, D. (2005). Patterns of parental authority and adolescent autonomy. In: Smetana, J. (ed.): New Directions for Child and Adolescent Development, V. 108 - Changing boundaries of parental authority during adolescence, pp. 5-16, San Francisco.

Block, J. (2008). Issues for DSM V: Internet adicction. Am J Psychiatry, 165:3 obtenido el 23.10.2013 de http://ajp.psychiatryonline.org/article. aspx?articleID $=99602$

Esteves, E., Murgui, S., Musitu, G. y Moreno, D. (2008). Relación entre el clima familiar y el clima escolar: el rol de la empatía, la actitud hacia la autoridad y la conducta violenta en la adolescencia. International Journal of Psychology and Psychological therapy, 9(01), pp. 123-136.

Estévez, A. Calvete, E. (2007). Esquemas cognitivos en personas con conducta de juego patológico y su relación con experiencias de crianza. Clínica y Salud, 18(1).

Esteves, E., Murgui, S., Musitu, G. y Moreno, D. (2008). Relación entre el clima familiar y el clima escolar: el rol de la empatía, la actitud hacia la autoridad y la conducta violenta en la adolescencia. International journal of Psychology and Psychological therapy, 9(01), pp. 123-136.

Fourment, K. (2009). Validez y confiabilidad del auto cuestionario de modelos internos de relaciones de apego (CaMir) en un grupo de madres de Lima Metropolitana. Tesis para licenciatura. Pontifica Universidad Católica del Perú.

Ipsos APOYO (2012). Informe del adolescente y el joven 2012. Informe gerencial de Marketing - IGM, Publicación virtual. Obtenido 22.10.2013 http://www.google. com.pe/url? sa $=\mathrm{t} \& \mathrm{rct}=\mathrm{j} \& \mathrm{q}=\& \mathrm{esrc}=\mathrm{s} \& \mathrm{frm}=1 \&$ source $=$ web\&cd $=1 \& \mathrm{ved}=0 \mathrm{CCg}$ QFjAA\&url=http\%3A\%2F\%2Fwww.ipsos-apoyo.com.pe\%2Fmarketingdataplus\% 2Fdownload_publi.php\%3Ffilename\%3D\%2FIgm\%2F2012\%2FIGM\%2520Perfil\% 2520del\%2520adolescente\%2520y \%2520joven\% 25202012.pdf\&ei =9yhnUpW9FPa w4APHrIG4DA\&usg =AFQjCNERWuoFPsGRYa8QD703gau1irmCxA

Matalinares, M. Díaz, G., Arenas, C., Dioses, A. Medina, N., Raymundo, O., Baca, D., Fernández, E., Uceda, J., Huari, Y., Villavicencio, N., Vargas, P. Quispe, M., Sánchez, E., Leyva, E. y Díaz, A. (2013) Adicción al Internet y agresividad 


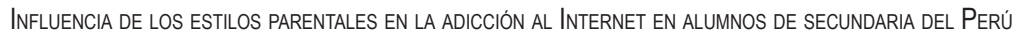

en estudiantes de secundaria del Perú. Revista de Investigación en Psicología de la UNMSM, 16 (1).

Melis F., Dávila M., Ormeño V., Vera, V., Greppi, C. y Gloger, S. (2001). Estandarización del P.B.I. (Parental Bonding Instrument) versión adaptada a la población entre 16 y 64 años del Gran Santiago. Revista Chilena Neuro-Psiquiátrica 2001; 39: 132-9.

Parker, G. Tupling, H. Brown, L. (1979). A parental bonding instruments. British Journal of Medical Psychology. 37 (52): 1-10.

Raymundo, O., Matalinares, M. y Baca, D. (2013) Propiedades psicométricas del test de adicción al Internet (TAI). Revista de Investigación en Psicología de la UNMSM, $16(1)$.

Raya, A. (2008). Estudio sobre los estilos educativos parentales y su relación con los trastornos de la conducta en la infancia. Tesis Doctoral, Universidad de Córdoba, Córdoba, España. Recuperado el 30 de Mayo de 2012 de http://helvia.uco.es/xmlui/ bitstream/handle/10396/2351/abre_fichero.pdf? sequence $=1$.

Vallejo, A., Osorno, R., Mazadiego, T. (2008). Estilos parentales y sintomatología depresiva en una muestra de adolescentes veracruzanos enseñanza e investigación en Psicología. Red de revistas científicas de América Latina, el Caribe, España y Portugal, 13(1), pp. 91-105.

Young, K. y Nabuco, C. (2011). Internet Addiction; a handbook and guide to evaluation and treatment. Canadá: Jhon Wiley y Sons, Inc. 